
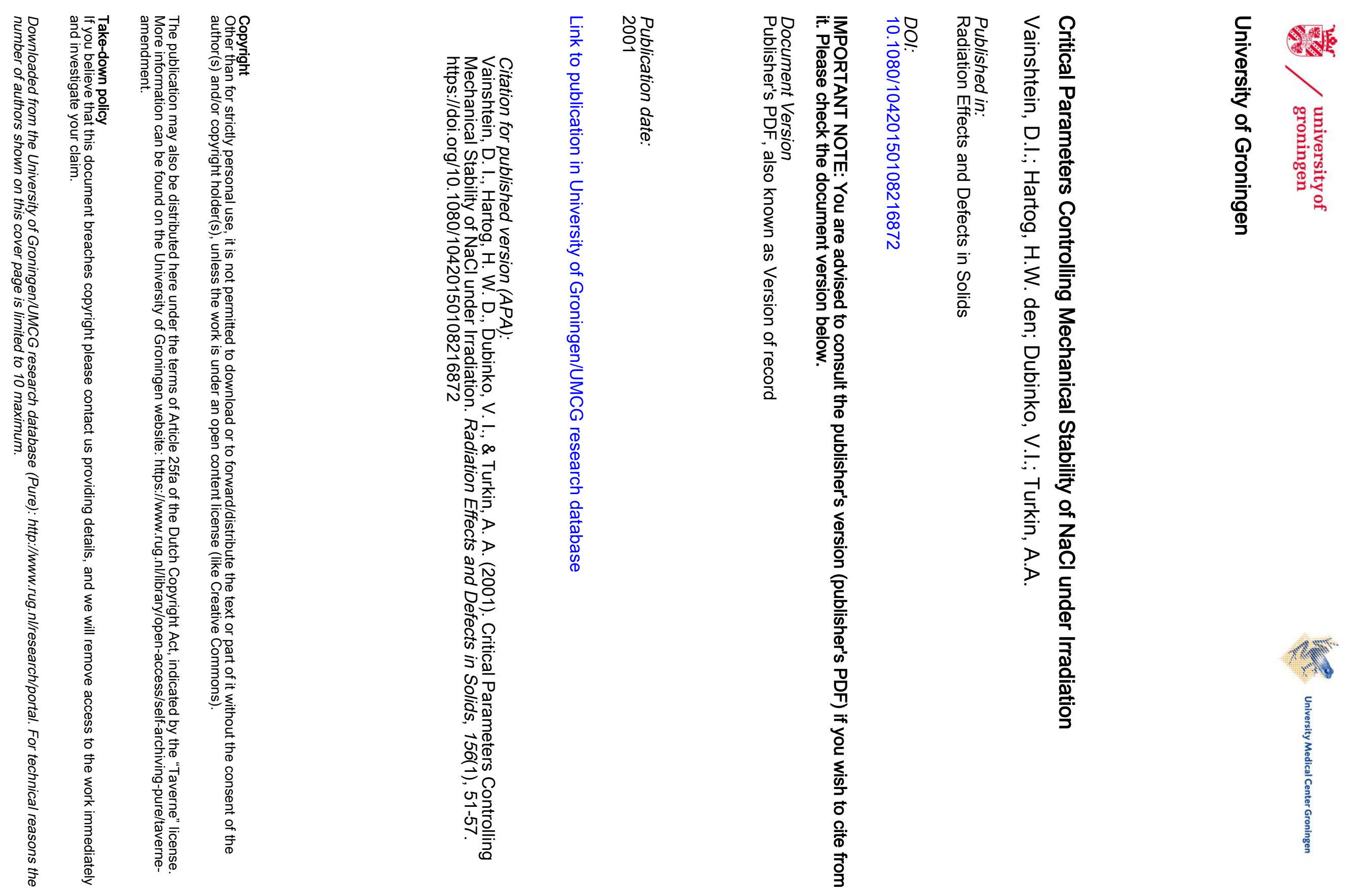


\title{
CRITICAL PARAMETERS CONTROLLING MECHANICAL STABILITY OF NaCI UNDER IRRADIATION
}

\author{
D. I. VAINSHTEIN ${ }^{\mathrm{a}}$, H. W. DEN HARTOG ${ }^{\mathrm{a}}$, V. I. DUBINKO \\ and A. A. TURKIN ${ }^{\mathrm{a}, \mathrm{b}}$ \\ ${ }^{a}$ Solid State Physics Laboratory, University of Groningen, 4 Nijenborgh, NL-9747 \\ AG Groningen, The Netherlands, ${ }^{\mathrm{b}}$ Kharkov Institute of Physics \& Technology, \\ 310108 Kharkov, Ukraine
}

(Received 7 April 2000; In final form 1 October 2000)

\begin{abstract}
A new concept of the radiation-induced microstructural evolution in ionic crystals has been verified experimentally by phenomena observed in heavily irradiated $\mathrm{NaCl}$, such as the formation of large vacancy voids followed by a sudden explosion-like fracture of crystalline samples. The new concept can be a prototype of an adequate description of the long-term behavior of important insulating materials in intense radiation fields, which can be employed for an evaluation of the critical effects expected under conditions of storage of high level nuclear waste and development of radiation resistant materials. In this paper, we present some new data on the microstructural parameters of materials doped with different impurities and summarize the critical microstructural, irradiation and material parameters that control mechanical stability of rock salt materials under irradiation.
\end{abstract}

Keywords: Radiation damage; Ionic crystals; Precipitates; Vacancy voids; Cracks.

\section{INTRODUCTION}

In the alkali halides, the principal radiation damage consists of bubbles of fluid halogen formed by agglomeration of $H$ centers and of the complementary inclusions of alkali metal ('colloids') formed by agglomeration of $F$ centers $[1,2]$. Our experiments on heavily irradiated pure, doped and

\footnotetext{
*Corresponding author. E-mail: H.W.den.Hartog@phys.rug.nl
} 
natural rock salt samples have shown that with increasing dose, the formation of relatively large voids was observed followed by a sudden fracture of the material [3] (Fig. 1). These phenomena could not be explained by the conventional Jain-Lidiard type models [1,2]. The difficulty appeared to be the creation of electroneutral vacancy pairs, since irradiation produced only Frenkel pairs in halide sub-lattice ( $H$ and $F$ centers). We have proposed a new model $[4,5]$, which involves the production of $V_{F}$ centers (a cation vacancy with a self-trapped hole) at dislocations as a result of their reaction with $H$ centers. Voids are shown to arise due to the formation of electroneutral vacancy pairs (two adjacent vacancies in the cation and in the anion sub-lattices) as a result of the reaction between $F$ centers and $V_{F}$ centers at the surfaces of halogen bubbles. The model predicts that voids grow to sizes exceeding the mean distance between colloids and bubbles, eventually absorbing them and, hence, bringing the halogen gas and metal to a back reaction. This leads to the explosive release of radiation-induced stored energy within the voids and to void-crack transformations [6]. We attribute the destruction of salt samples after high dose irradiation to these phenomena. In this paper, we present some new data on the microstructural

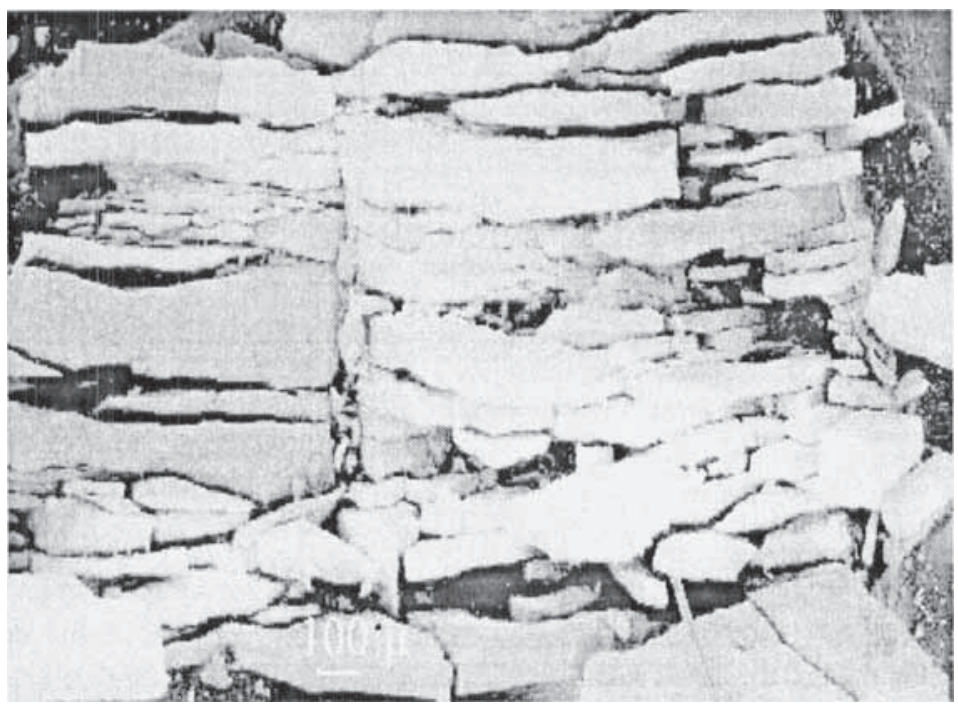

FIGURE 1 Explosive fracture of $\mathrm{NaCl}+\mathrm{K}(0.1 \mathrm{~mol} \%)$ samples irradiated with $0.5 \mathrm{McV}$ electrons to $300 \mathrm{Grad}$. SEM micrograph of the exploded sample reveals large fragments and dust-like particles. 
parameters of materials doped with different impurities and summarize the critical microstructural, irradiation and material parameters that control mechanical stability of rock salt.

\section{SODIUM AND CHLORINE PRECIPITATES}

The volume fraction of chlorine 'bubbles' (or rather solid or liquid chlorine precipitates, since the pressure in them is too high for the liquid-gas transition) is closely related to that of sodium colloids (Fig. 2), which is a measure of the energy stored by irradiation. The higher it grows, the more potentially unstable the material becomes. But the actual mechanical stability is determined by a scenario of the stored energy release rather than by its value. If the back reaction quantum is small enough (as it is assumed in the Jain-Lidiard model, which considers only the recombination between

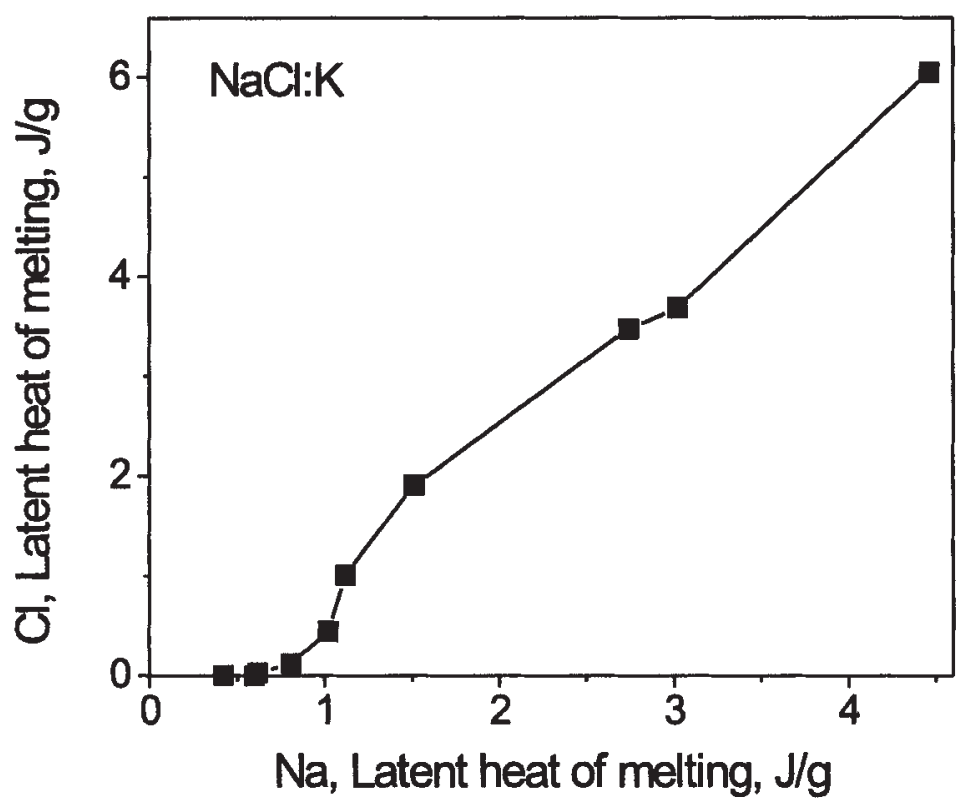

FIGURE 2 Latent heat of melting of chlorine (which is proportional to the chlorine bubble volume fraction) vs. the latent heat of melting of sodium (which is proportional to the sodium colloid volume fraction). The linear dependence starts when a critical volume fraction of chlorine is reached resulting in its aggregation in bubbles. 
chlorine molecules and $F$ centers) then it may just limit the stored energy accumulation. But the voids can collect a large number of chlorine molecules before the collision with colloids starts, which changes the scale of the back reaction from the atomic to the macroscopic one, resulting in material fracture.

The bubble number density, which controls the critical irradiation dose of their transition to voids, is an important parameter. It is expected to depend on the content of impurity ions that can act as the bubble nuclei. It can be seen that if the bubble number density is higher than some threshold value, the void formation can be suppressed. in which case the release of stored energy is possible only by direct collisions between growing sodium and chlorine precipitates. The latter process may be more gradual due to a finer scale of the mechanism involved, which may be less dangerous for the mechanical stability of the material. So it may be an attractive type of further research to find the dopants acting as efficient traps for $I /$ centers, which could disperse chlorine among finer bubbles.

Another critical parameter is the colloid number density, which is shown in the companion paper [6]. If it is below $10^{21} \mathrm{~m}^{-3}$, then the void-crack transition size is large and difficult to reach. If it is above $10^{22} \mathrm{~m}^{-3}$, then a high colloid volume fraction is required to initiate the void-crack transition, which progresses gradually and may not result in explosive fracture. The materials doped with a majority of investigated dopants (such as $\mathrm{Ba}$ or $\mathrm{K}$ ) and natural rock salt samples show the transitional behavior, which is the most susceptible to explosive fracture. This picture is consistent with a comparative analysis of the colloid number densities measured in different materials (Fig. 3) according to which the colloid number density gradually increases from materials doped with $\mathrm{Br}$ to those doped with $\mathrm{KBF}_{4}$.

\section{VOIDS}

Voids have been shown to arise from halogen bubbles and grow to sizes exceeding the mean distance between colloids and bubbles, eventually absorbing them, and, hence, bringing the large numbers of halogen gas and metal atoms to a back reaction within the voids. Thus, the void size is a parameter of primary importance that should be monitored during irradiation, since the unstable materials produce the largest voids (Fig. 4). 


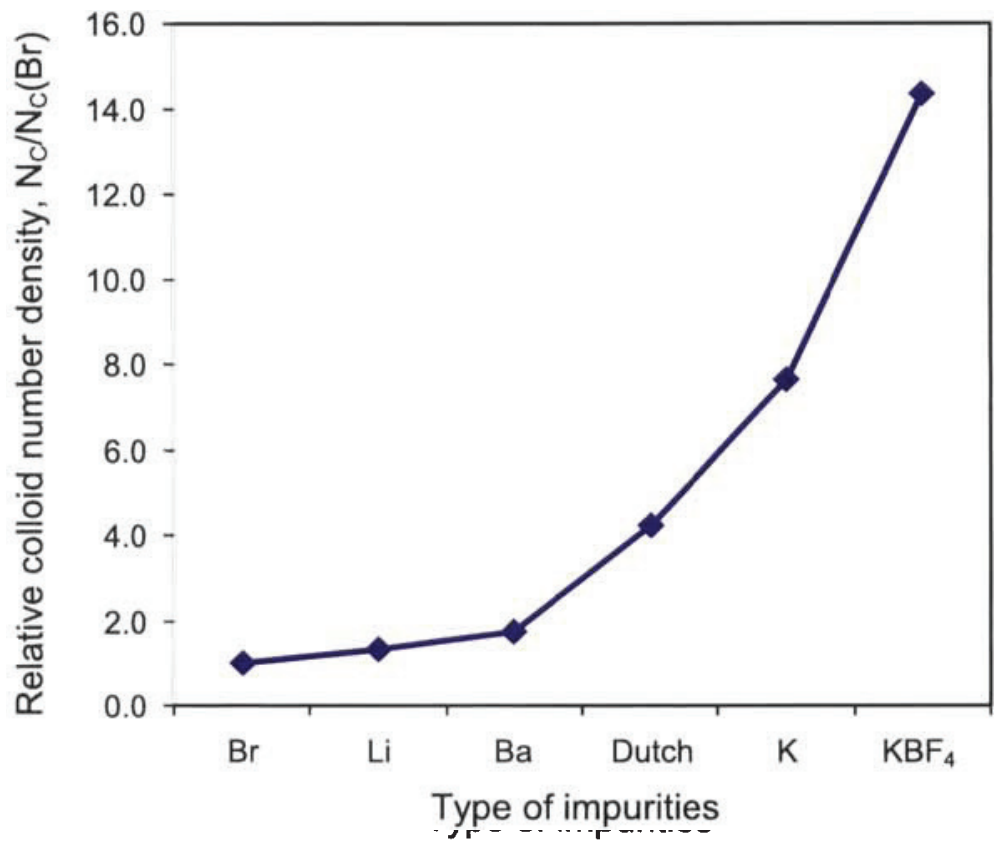

FIGURE 3 Colloid number densities in different materiais including Dutch rock salt after $300 \mathrm{Grad}$ irradiation obtained by measuring the ESR signals from sodium colloids. A signal from $\mathrm{Br}$ doped samples was selected as a reference point.

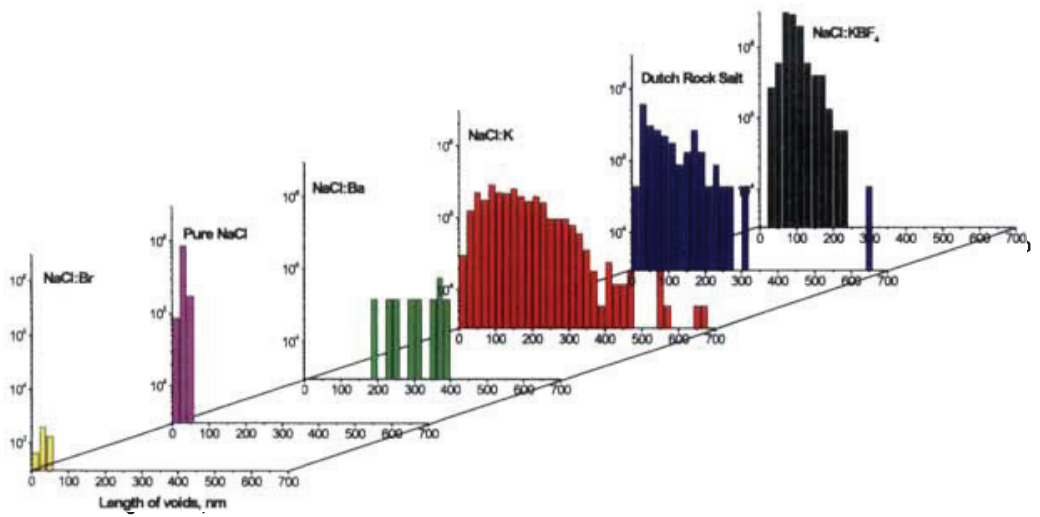

FIGURE 4 Comparison of void size distributions formed in different materials irradiated up to $300 \mathrm{Grad}$ at temperatures ranging from $60^{\circ} \mathrm{C}$ to $130^{\circ} \mathrm{C}$. The most unstable materials are natural rock salt and $\mathrm{NaCl}$ doped with $\mathrm{K}$, in which the largest voids are produced. 
Another important factor is the shape of the voids, which shows at what stage of radiolysis the material is. If the voids are equiaxial then the explosive back reaction has not yet started or it is too weak to cause the voidcrack transition. The appearance of elongated (or penny-shaped) voids/ cracks indicates the beginning of the explosive fracture process (see the companion paper [6]).

\section{IRRADIATION DOSE VS. MATERIAL PARAMETERS EFFECTS}

We have shown that the microstructural evolution in irradiated rock salt is characterized by critical stages such as the formation of sodium precipitates and chlorine 'bubbles' followed by the bubble-void transition and a subsequent void-crack transition leading to explosive fracture of the material. Each stage begins after some critical irradiation dose, which can vary enormously for different materials and irradiation conditions. This is illustrated in Figure 4, which shows that voids of very different sizes are formed in different materials by the same irradiation dose. Naturally, the dose required to initiate the explosive back reaction in voids depends on the material parameters, such as the impurity type and content, which can be used in the development of radiation resistant materials.

Our experimental data obtained under laboratory conditions are well described in terms of the critical parameters given by the present theory. So they should be regarded as a first demonstration of possible scenarios of the rock salt behavior under irradiation rather than as quantitative predictions of the backfill material behavior under storage conditions. The latter requires further and more detailed investigations.

\section{Acknowledgements}

This study was supported by the Dutch Ministry of Economic Affairs and by the NATO Linkage Grant CRG.LG 973314.

\section{References}

[1] Jain U. and Lidiard, A.B. (1977), Phil. Mag. 35, 245.

[2] Soppe, W.J., Donker, H., Garcia Celma A. and Prij, J. (1994). J. Nucl. Mater. 217, 1. 
[3] Vainshtein, D.I., Dubinko, V.I., Turkin A.A. and den Hartog, H.W. (1999). Radiation Effects and Defects in Solids 150, 173.

[4] Dubinko, V.I., Turkin, A.A., Vainshtein D.I. and den Hartog, H.W. (1999). J. Appl. Phys. 86, 5957.

[5] Dubinko, V.I., Turkin, A.A., Vainshtein D.I. and den Hartog, (2000). J. Nucl. Mater: 227, 184 .

[6] Dubinko, V.I., Turkin, A.A., Vainshtein D.I. and den Hartog, H.W. (2001). Radiation Effects and Defects in Solids, in press. 